\title{
Density-Based Traffic Control System for Emergency Vehicles using Artificial Intelligence
}

\author{
Priyanka Abhang, Vinit Agrharkar, Shriya Akella, Siddhant Bhagat, Shrishti Kaushik, Piyush Mishra, \\ YS Rao
}

\begin{abstract}
Fire brigade officers, health care personnel, police are often delayed due to traffic congestion, across major cities in India. Considering the predicament, Artificial Intelligence has the potential to enable us to solve such problems by adopting a number of unique perspectives and approaches, especially in this domain. The solution developed by us enables an emergency vehicle driver to select the route to reach the destination as quickly as possible. As cameras are deployed at most of the traffic signals today, especially in cities where traffic congestion is a major pain point, Video Analytics can be used for calculating vehicle count, which will be streamed and updated continually. We create effective algorithms to alter the time of the traffic signals based on this real-time vehicle count, the distance of the vehicle from the signal, the bearing angle made by the vehicle with the signal and also by making sure that the traffic congestion doesn't increase exponentially and multiple emergency vehicles do not put the system in a deadlock. The loss of life due to accidents and the delay in getting the required treatment must be avoided. The designed system will automatically control traffic light intervals based on vehicle density. This solution will allow an emergency vehicle to reach its destination during emergencies, plying on the best possible route, in the most decongested traffic conditions, which will be facilitated by specifically developed algorithms. To save human life from accidents and unnecessary delays due to traffic congestion, is the main aim of our system.
\end{abstract}

Keywords: Anti-collision algorithm, Computer Vision, Realtime location monitoring, Video Analytics

\section{INTRODUCTION}

According to a study, it is found that India is the only country with the death toll of more than 15 deaths and 53 injuries per hour due to road accidents. In fact, one person dies every 4 minutes in India owing to this cause.

Emergency vehicles play an important role in every lifethreatening situation.

Revised Manuscript Received on June 15, 2020.

* Correspondence Author

Priyanka Abhang*, Student, Department of Electronics and Telecommunications, S.P.I.T, Mumbai, India.

Vinit Agrharkar, Student, Department of Electronics and Telecommunications, S.P.I.T, Mumbai, India.

Shriya Akella, Student, Department of Electronics and Telecommunications, S.P.I.T, Mumbai, India.

Siddhant Bhagat, Student, Department of Electronics and Telecommunications, S.P.I.T, Mumbai, India.

Shrishti Kaushik, Student, Department of Electronics and Telecommunications, S.P.I.T, Mumbai, India.

Piyush Mishra, is currently a final year student in pursuing Electronics and Telecommunications Engineering at S.P.I.T, Mumbai. His research interests include Data Science, Machine Learning and IOT.

Dr. Y.Srinivasa Rao, Vice-Principal, Department of Electronics and Telecommunications Engineering Sardar Patel Institute of Technology

(C) The Authors. Published by Blue Eyes Intelligence Engineering and Sciences Publication (BEIESP). This is an open access article under the CC BY-NC-ND license (http://creativecommons.org/licenses/by-nc-nd/4.0/)
Traffic jams take more than $20 \%$ of patients' lives in an ambulance but when the patient's condition is very serious the percentage of patient death is increased [1]. Similar statistics support the state of fire- related emergencies, crime-related events and many more realms in India.

Consider a singular example of how grave the problem can turn to in India. In an official release by the Management of Acute Coronary Event (MACE) Registry of the Indian Council of Medical Research (ICMR) in 2017, over $50 \%$ of those who suffer from a heart attack in India, reach hospital late. The ideal duration defined by doctors is 30 minutes, but every second person in India reaches hospital around 400 minutes later after the occurrence of a cardiac arrest. This is just one of the many cases which occur on the Indian roads each day. The major reason attributed to this delay is the ever-increasing vehicular traffic on the Indian roads. The framework to reduce the intensity of vehicular traffic has been proposed in a variety of different ways, but those are solutions which would need years of implementation and face bridled implementation efficiency concerns. Hence, our paper discusses the viability and technical scope of an automated and integrated traffic management infrastructure, specifically to facilitate shorter response times for emergency vehicle services; and, consequently, reducing the number of lives lost due to the issues discussed. This paper aims to add value to traffic research communities, the traffic police and other stakeholders which are developing solutions to solve similar problems.

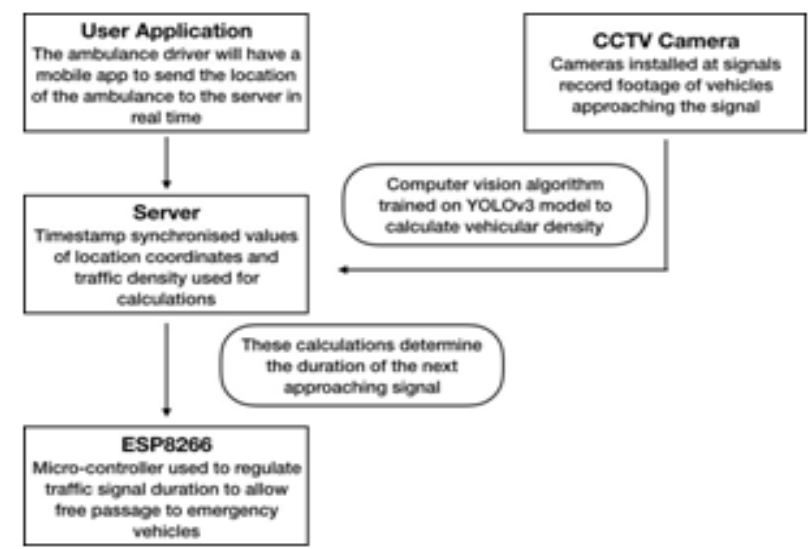

Fig. 1. Flowchart of proposed system

The influx of rising traffic is an ineluctable condition in metropolitan cities, this issue needs to be addressed with smart solutions like the smart traffic management system integrated with video analytics. The Smart Traffic Management System visualizes the movement of emergency vehicles using video analytics.

Published By:

Blue Eyes Intelligence Engineering DOI: 10.35940/ijeat.E1228.069520

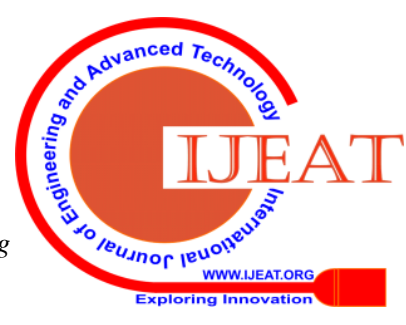




\section{Density-Based Traffic Control System for Emergency Vehicles using Artificial Intelligence}

The system is designed to control the traffic signals along the emergency vehicle's travel path. It essentially tracks the position of the priority vehicles relative to the incoming traffic signals in its path. The objective of our solution is to control the traffic signal duration/ intervals on the basis of:

1) a threshold value of traffic density

a threshold value of the distance and angle between the emergency vehicle and the next approaching signal

The duration of the signal can be altered on the case-tocase basis, thus ensuring priority to the emergency vehicles, and at the same time, not wholly disrupting regular traffic. The system will be equipped with a collision detection algorithm in case of a multi-vehicular emergency to avoid collision by defining all the disparate cases of a collision at a junction. The algorithm used for object detection is YOLOv3 on COCO dataset [2]. It helps us to perfectly detect number of vehicles in a frame and calculate the vehicular density accordingly.

\section{LITERATURE SURVEY}

The most important parameters that one needs to consider while assessing the state of traffic are: traffic volume, speed, and density. Traffic volume and speed have been measured by existing loop-detector based surveillance systems. However, with the newer methods of research in the domain of computer vision, accurate models have been developed and tested to calculate traffic density accurately. Chung and Keemin, in [3] have developed a deep Convolutional Neural Network method which was operated on video images. The experimental results showed the outperformance of their model over existing approaches in practice.

Traffic lights are the only means to regulate vehicular density on roads. Keeping signal duration static is not the most efficient way to control vehicle movement smoothly, especially on congested roads. Abu Zaid et. al. [4] proposed a methodology for controlling traffic signal duration as a function of the number of vehicles in the proximity of a particular signal. The duration for each light was calculated based on operations using Artificial Neural Networks and Fuzzy logic on image data extracted from a captured video. The results generated showed promise to regulate traffic flow and reduce the waiting time wasted in roads.

Video Monitoring and Surveillance Systems have been an integral part of the continuous development cycle of "Smart Traffic lights". Anurag et. al. [5] developed a method which helps realize real-time traffic density-based smart traffic light implementation. The paper elaborated on a method where live video feeds from cameras installed at traffic junctions was processed to determine optimum signal duration for faster movement of traffic in congested spaces. Another crucial use case for the utilization of Computer Vision methods for enabling the implementation of smart traffic lights, is for providing "free" (devoid of congestion, or having minimal congestion) corridors for the fast movement of emergency vehicles. Proposed framework to reduce the latency of emergency services for vehicles, and varying related driving

policies and drivers' behavior, has been discussed by the authors of [6].

This paper talks about a real-time traffic adaptive signal control system which is called RHODES. The RHODES approach takes in an input data and controls the traffic problem by decomposing it into sub parts in a hierarchical pattern. This pattern is analyzed, optimized and a data structure and computer/communication approach is used to make decisions. This paper presents a simulation of various scenarios and demonstrates the robustness of the system.

A wireless communication using Vehicular Ad HocNetwork(VANET) has been proposed in this paper [8]. It enables various vehicles to communicate amongst themselves as well as road side units. The Intelligent Traffic Lights (ITL) system relays updates regarding the traffic conditions. The ITLs are responsible for collecting information regarding road conditions and updating the user from time to time. Ad hoc On- Demand Distance Vector(AODV) is used as a routing protocol in VANETS. The AODV sends messages regarding traffic conditions to the nearest ITL. The throughput for various number of nodes has been calculated in the results. This system has minimum delay and also a good throughput value.

In the system developed by us, we have planned to use adequate hardware support to make our system better suited for real-time data processing needs. It is imperative that we discuss the work of Jun Zhang et. al [9] in the related field. The work done by them was primarily focused on streamlining the bus-scheduling process, using passengers' smart card and associated GPS data. Passenger flow estimation using the above-mentioned data points was carried out, resulting in high performance against the metrics: prediction-estimation proximity and origindestination accuracy, carried out on passenger-level granularity of data.

A large number of studies have been published to generate point forecast type of predictions. However, prediction interval generation has garnered much attention to quantify the uncertainty associated with point forecasts. This was the central focus of the work Wei Huang et. al, in their work [10]. The paper focuses on second order moment modelling emerging from conventional short-term traffic condition prediction. When contrasted with the performance of an offline model based on GARCH methodology, this paper proposed an online OSAF + AKF approach of predicting in real- time the seasonal heteroscedasticity in traffic flow series. This paper performed operations on a 15minute interval dataset, which can be modified to accommodate different intervals as per the needs.

\section{WORKING}

When an emergency vehicle starts its journey the GPS module in the vehicle starts calculating the distance and the bearing of the vehicle from the Next Approaching Signal (NAS).

The video camera at the signal helps to take into consideration information regarding vehicular density.

Published By:

Blue Eyes Intelligence Engineering

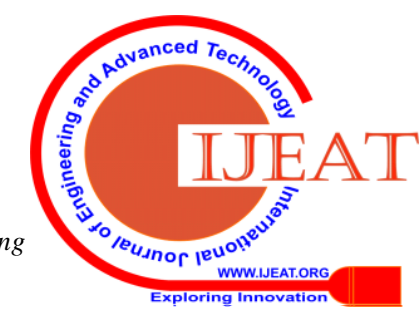

\& Sciences Publication

(C) Copyright: All rights reserved. 


\section{Density-Based Traffic Control System for Emergency Vehicles using ArtificialIntelligence}

These parameters are then fed into the microcontroller which then makes a decision regarding the amount of time the signal will turn green. After this, the system resumes normal operation. The anti-collision algorithm makes sure that in any case two emergency vehicles do not collide.

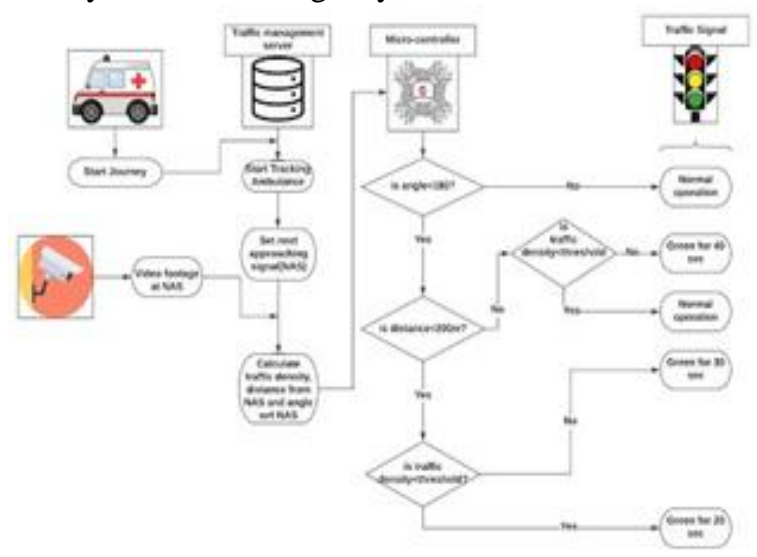

Fig. 2. Use case of the working

\section{ALGORITHM}

The CCTV surveillance cameras capture the real-time footage from all the signals and forward them to the Traffic Management server (a centralized server which keeps a track of the location and current state of every traffic signal within a predefined geographical area). The server employs a machine- learning algorithm to detect the density of the vehicles on the road (within the range of the camera under operation), distance from the next approaching signal (NAS) for the emergency vehicle, angle the vehicle (geographical bearing) makes from the NAS and tracks its path. We use the 'haversine' formula to calculate the great-circle distance between two points (expressed as geographical coordinates)

- that is, the shortest distance over the earth's surface, colloquially called the "crow- fly" distance.

$$
\begin{aligned}
& \mathrm{a}=(\sin (\delta \phi / 2)+\cos \phi 1 \cdot \cos \phi 2 \cdot \sin \delta \phi / 2) \\
& (1) \mathrm{c}=2 \operatorname{atan} 2((\mathrm{a},(1 \mathrm{a}))(2) \\
& \mathrm{d}=\mathrm{Rc}(3)
\end{aligned}
$$

Where $\phi$ is latitude, $\lambda$ is longitude, $\mathrm{R}$ is earth's radius (mean radius $=6,371 \mathrm{~km}$ ); note that the angles need to be in radians to pass to the trigonometric functions.

Using the principles of forward azimuth and orthodrome (great circle path) assumptions, we use the following formulae to calculate bearing between two latitudes.

$$
\theta=\operatorname{atan} 2(\sin \delta \Lambda \cdot \cos \phi 2, \cos \phi 1 . \sin \phi 2 \sin \phi 1 . \cos \phi 2 \cdot \cos \delta \lambda 1)
$$

Where $\phi 1, \lambda 1$ is the start point, $\phi 2, \lambda 2$ the end point $(\Delta \lambda$ is the difference in longitude).

The model detects the emergency vehicle by making bounding boxes around it and correctly classifying them. Once the number of emergency vehicles in a frame is calculated and the area of the frame is measured, the vehicular density is calculated without taking into consideration the empty spots on the road. Next, the distance and the angle of the emergency vehicle from the NAS is calculated. Once all these three parameters are calculated for a particular timestamp, they are sent to the microcontroller (NodeMCU). The data collection module, the microcontroller gathers all the parameters and analyses the data. The NodeMCU implements the proposed algorithm and sends its decision to the traffic signal. The decision executes the duration and sequence of the signal as per the algorithm developed as seen in Table I. After the passage of an emergency vehicle, the system goes back to its normal operation

A very important consideration in this proposed system is that of an anti-collision algorithm to prioritize amongst emergency vehicles in case of their simultaneous arrival. We now discuss a few use cases and how our algorithm works in those cases in Table II:

Case 1: Considering two or three different kinds of emergency vehicles approach a junction at the same time, in this scenario priority will be given on the basis of size of the vehicle and time required for accelerating and decelerating it. In case of fire brigades because of their massive load, it becomes difficult to slow them down and stop at a traffic signal and then pick up speed again which would cause them delay in reaching the destination as well as to the emergency vehicles next in line. Thus, they are allowed to cross first without having to stop at the signal followed by ambulances and then police vans. This would result in efficient management of time and could save lives. Case2: Ambulances are one of the most ubiquitous emergency vehicles and requires great attention due to both its importance and common occurrence. Hence, we discuss their priority separately, in case 4 . For case 2, we aim to consider all the emergency vehicles except the ambulance. In such a scenario, usually the emergency vehicles are required to be tracked only once i.e en route to the emergency. The other way around is not of great significance in the above-mentioned case. Hence, the best way of allotment of priority would be by the means of FCFS(first-come-first-serve) method. The vehicle which logs in earlier in our system would be given priority over the vehicles which log in later. This would ensure an efficient management of emergency situations and would ease complexities of our proposed solution. Case3: An extension of Case 2 would be the consideration that both the emergency vehicles start their journey at the same time. This would require us to prioritize the vehicles in a more efficient way. So, the priority, in this case, will be decided in a clockwise fashion. Case 4: In case of ambulances, it is essential to manage traffic both ways i.e when the ambulance is on its way to pick

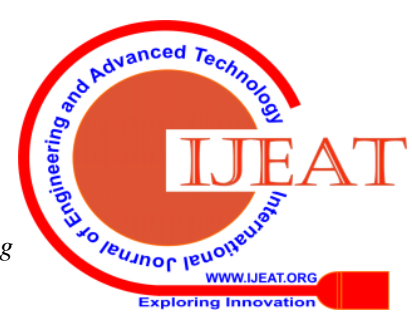


Density-Based Traffic Control System for Emergency Vehicles using Artificial Intelligence

Table- I: Algorithm

\begin{tabular}{llll}
\hline TrafficDensity & Distance & BearingAngle & Output \\
\hline Morethanthreshold & Lessthanthreshold & Lessthan180 & Green for 30s \\
Morethanthreshold & Morethanthreshold & Lessthan180 & Green for 40s \\
Lessthanthreshold & Lessthanthreshold & Lessthan180 & Green for 20s \\
Lessthanthreshold & Morethanthreshold & Lessthan180 & NormalOperation \\
NA & NA & More than 180(vehicle Normal Operation & \\
& & crossedsignal) & \\
\hline
\end{tabular}

up a patient from the emergency location and when it is taking a patient to the hospital. This scenario occurs only in this case as for other emergency vehicles like fire brigades and police vans, the journey back to the station is not an emergency situation. Thus, priority is given to the ambulance which is on its way to pick up a patient because ambulances have medical professionals and life support systems on-board which would help in stabilizing the patient's condition who is on the way to the hospital whereas the other one has no such facility present with him/her thus requires immediate medical attention

Table- II: Anti-collision Algorithm

\begin{tabular}{ll}
\hline Case & Priority \\
\hline Case & Police Van $<$ Ambulance $<$ Fire Brigade \\
1 & \\
Case & Police Van (at $\mathrm{t})>$ Police Van $\left(\right.$ at $\left(\mathrm{t}+\mathrm{t}^{*}\right)$ ) \\
2 & \\
Case & Sequentially (one at a time) \\
3 & \\
Case & Ambulance (towards patient) $>$ Ambulance \\
4 & (towards hospital) \\
\hline
\end{tabular}

\section{RESULTS AND DISCUSSIONS}

These are the results that were simulated by our prototype algorithm, as shown in Fig. 3.

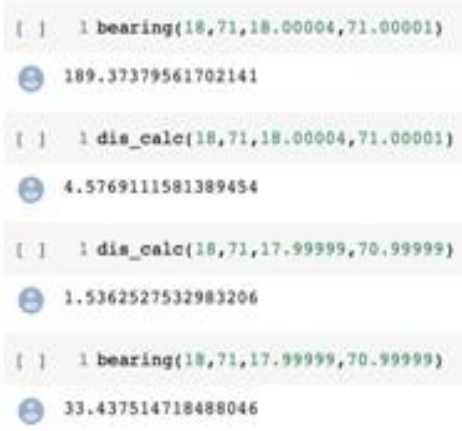

Fig. 3. Calculation of bearing and distance

The calculated geographical bearing and the distance of the emergency vehicle from the NAS plays a significant role in determining the traffic light sequence and duration, that is for how long different signal lights will be switched on or off. The part of our system which performs vehicle detection using computer vision was already discussed in the previous section, with output shown in fig. 4., fig 5., fig 6 . and fig 7 . In the above output screen snapshot, the function bearing() returns the azimuthal angle between the two coordinates passed as comma-separated parameters. The function dis calc() returns shortest distance along earth's surface for the parameters passed to the function.

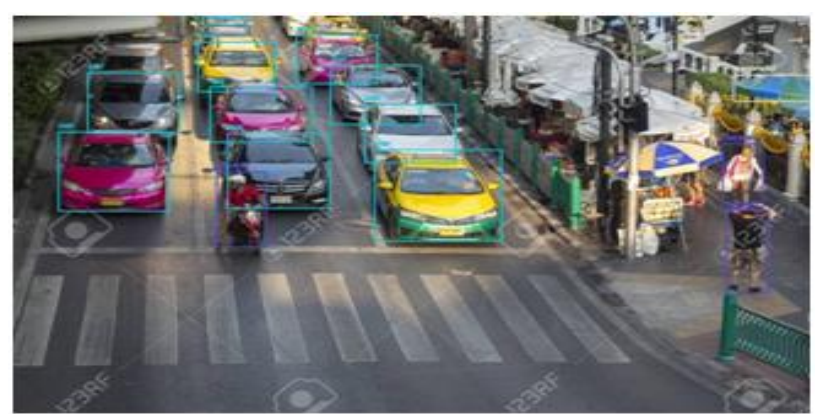

Fig. 4. Sample output for vehicle detection

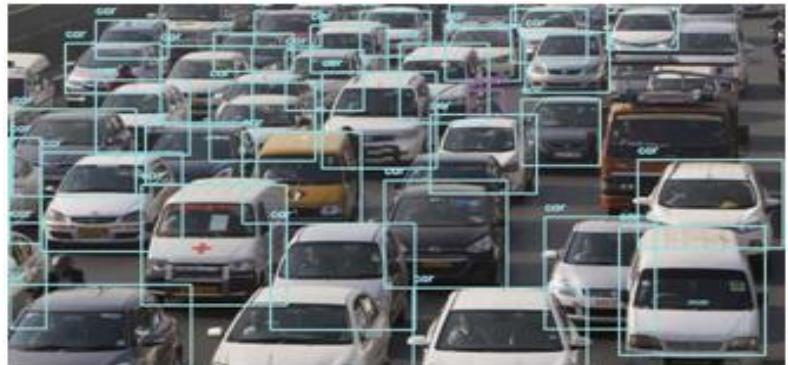

Fig. 5. Sample output for vehicle detection

The proposed system could be used to regulate traffic according to density at all places to ensure lesser traffic jams and video analytics could be utilized to read license plates to help curb theft. It could also be used for better security of specific vehicles' analysis of the real-time movement of that particular vehicle (asset-tracking) could be performed. From a longer-term perspective, we can implement homogeneous emergencies. For making the project viable, we have used a lean hardware approach and utilized software capabilities to deliver efficiency. The potential impact of the proposed work is substantial, being effective, accurate and inexpensive, it can be applied in practice

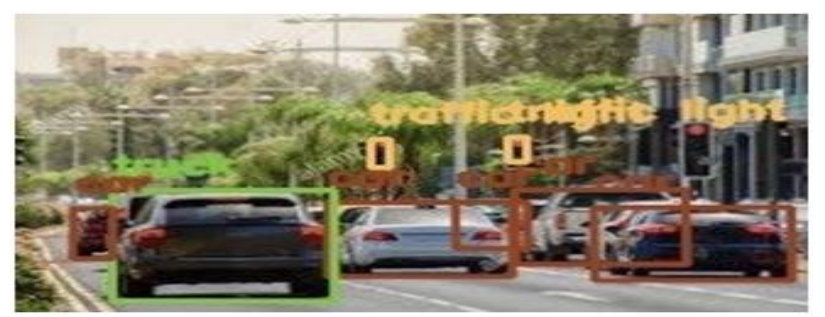

Fig. 6. Sample output for vehicle detection

Published By:

Blue Eyes Intelligence Engineering

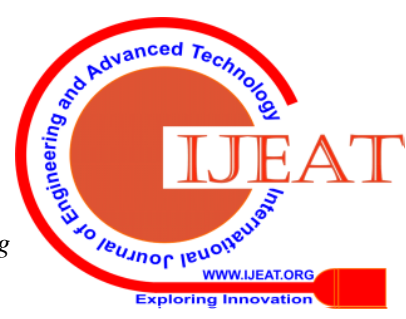




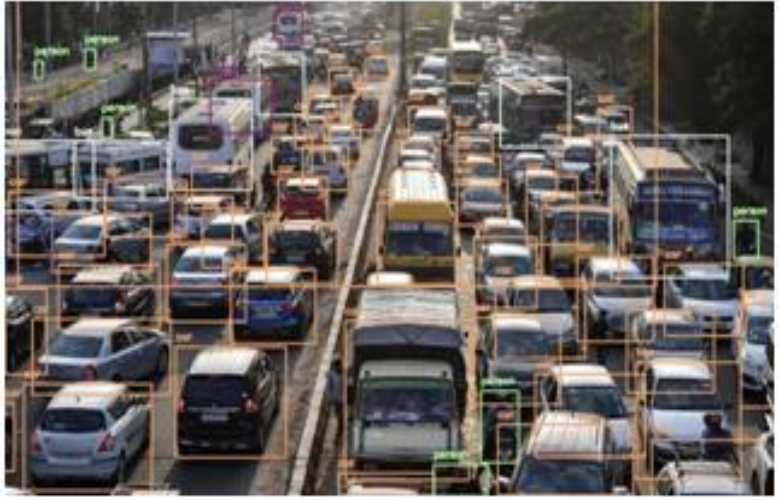

Fig. 7. Sample output for vehicle detection

lane traffic monitoring for ensuring better road safety and lesser traffic. Once the system is in place, we can develop trends for historical traffic patterns, hence providing valuable insights to the traffic police. It can also be used for helping the concerned authorities with pothole repair work, we can identify location of potholes (with minor modifications of the cameras) and relay the same information to them timely.

\section{CONCLUSION}

The system encapsulates vehicle's information with the use of object detection algorithms. It helps in understanding traffic management systems and drastically condenses the transit delay that is prevailing in the cities. It detects and counts the number of emergency vehicles that are approaching the signal in real time and alters the signal time to ensure effective congestion handling. The experimental results show that the parameters taken into consideration are suitable for real-time applications as they are precise and fast responsive. We had discussed a number of cases for handling collision which might arise in our system. Although we have evaluated a number of relevant parameters to ensure sound decision- making, there can be some additional features, like, intra- vehicular communication, and incorporation of additional data points for better understanding the nature of

\section{REFERENCES}

1. H. K. Kanzaria, M. A. Probst, and R. Y.Hsia, "Emergency department death rates dropped by nearly 50 percent," Health Affairs, vol. 35, no.

2. O. Masurekar and O. Jadhav, "Prateek Kulkarni and Shubham Patil "Real Time Object Detection Using YOLOv3," International Research Journal of Engineering and Technology (IRJET), 2020.

3. J. Chung and K. Sohn, "Image-Based Learning to Measure Traffic Density Using a Deep Convolutional Neural Network," IEEE Transactions on Intelligent Transportation Systems, vol. 19, no. 5, 2018, pp. 1670-1675. [Online]. Available: 10.1109/tits.2017. 2732029;https://dx.doi.org/10.1109/tits.2017.2732029

4. A. Zaid, Y. Suhweil, and M. A. Yaman, "Smart controlling for traffic light time," in Proc. IEEE Jordan Conf, 2017, pp. 1-5.

5. Kanungo, A. Sharma, and C. Singla, "Smart traffic lights switching and traffic density calculation using video processing," in Proc. Recent Adv, 2014, pp. 1-6.

6. S. Djahel, M. Salehie, I. Tal, and P. Jamshidi, "Adaptive traffic manage- ment for secure and efficient emergency services in smart cities," in Proc. IEEE Int. Conf. Pervasive Comput. Commun. Workshops (PERCOM Workshops), 2013, pp. 340-343.

7. P. Mirchandani and L. Head, "A real-time traffic signal control system: Architecture, algorithms, and analysis," Transp. Res. C, Emerg. Technol, vol. 9, 2011, pp. 415-432.

8. G. S. V. Khekareanda and Sakhare, "A smart city framework for 7, 1997, pp. 1303-1308.

intelligent traffic system using VANET," in Proc. Int. Mutli-Conf. Automat., Comput., Commun., Control Compressed Sens. (iMac4s), 2013, pp. 302-305.

9. J. Zhang, "A real-time passenger flow estimation and prediction method for urban bus transit systems," IEEE Trans. Intell. Transp. Syst, vol. 18, no. 11, 2017, pp. 3168-3178.

10. W. Huang, "Real-time prediction of seasonal heteroscedasticity in vehicular traffic flow series,'

11. IEEE Trans. Intell. Transp. Syst, vol. 19, no. 10, 2018, pp. 31703180 .

\section{AUTHORS PROFILE}

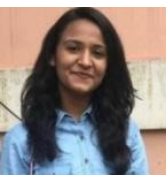

Priyanka Abhang is currently a final year student in the Electronics and Telecommunications Department of S.P.I.T, Mumbai. Her research interests include Machine Learning, Cyber Security, and Embedded Systems.

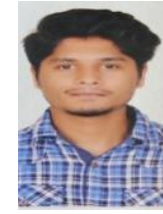

Vinit Agrharkar is currently a final year student in the Electronics and Telecommunications Department of S.P.I.T, Mumbai. His research interests include Machine Learning, Cyber Security and IOT.

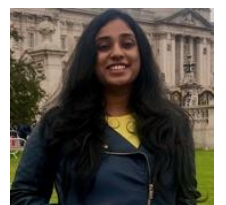

Shriya Akella is currently a final year student in the Electronics and Telecommunications Department of S.P.I.T, Mumbai. Her research interests lie in Machine learning, Computer Vision, Data Analytics and Embedded Systems.

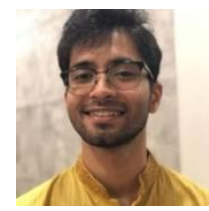

Siddhant Bhagat is currently a final year student in the Electronics and Telecommunications Department of SPIT, Mumbai. Her research interests lie in Machine learning, Computer Vision, Data Analytics and Embedded Systems.

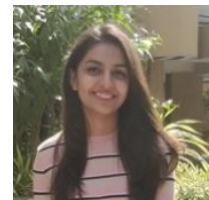

Shrishti Kaushik is currently a final year student in the Electronics and Telecommunications Department of S.P.I.T, Mumbai. Her research interests include Data Science, Machine Learning and Artificial Intelligence.

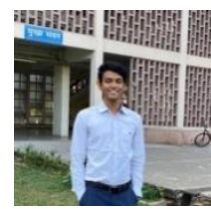

Piyush Mishra is currently a final year student in pursuing Electronics and Telecommunications Engineering at S.P.I.T, Mumbai. His research interests include Data Science, Machine Learning and IOT.

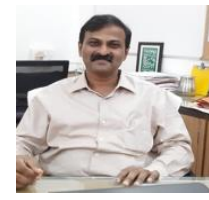

Dr. Y.Srinivasa Rao holds a PhD degree from IITBombay and has a work experience of more than 10 years in the industry. He has been in the teaching profession for over 27 years, of which he has been associated with Bhartiya Vidya Bhavans' Sardar Patel College of Engineering and subsequently, Sardar Patel Institute of Technology for more than 19 years. He has served as the Head of Department several times and is currently the Vice-Principal as well as the Dean of R\&D at Sardar Patel Institute of Technology

Published By:

Blue Eyes Intelligence Engineering DOI: 10.35940/ijeat.E1228.069520

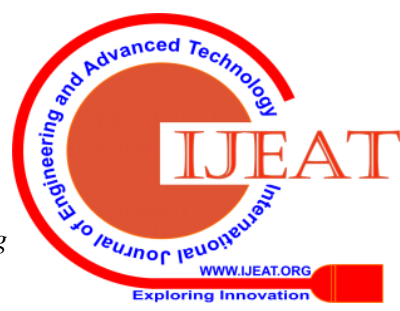

